\section{GP256 IMPACT OF A MEDICATION SAFETY BUNDLE ON PHARMACEUTICAL CARE ISSUES IN A NEONATAL INTENSIVE CARE UNIT (NICU)}

\footnotetext{
${ }^{1,2}$ Ann-Marie Cassar Flores, ${ }^{3,4}$ Naomi McCallion, ${ }^{3}$ Nurul Aminudin, 1,2Brian Cleary*. ${ }^{1}$ Pharmacy Department, Rotunda Hospital, Dublin, Ireland; ${ }^{2}$ School of Pharmacy, RCSI, Dublin, Ireland; ${ }^{3}$ Department of Paediatrics, Rotunda Hospital, Dublin, Ireland; ${ }^{4}$ Department of Paediatrics, RCSI, Dublin, Ireland
}

\subsection{6/archdischild-2019-epa.315}

Introduction Neonatal intensive care units are high risk areas for medication errors. The complexity of the medication use process for high-alert medications in sick neonates leads to multiple opportunities for error. A medication safety bundle was designed to assist with the prescribing, preparation and administration of four high alert continuous infusions - HACIs (morphine, dopamine, insulin and heparin), with the aim to reduce medication errors. Pharmaceutical care issues (PCIs) identified before and after the implementation of the medication safety bundle were compared.

Method The bundle of medication safety strategies was implemented in a level III NICU in Dublin. The medication safety bundle involved implementing: (i) Standard concentration infusions and drug monographs- this replaced the process of diluting medications using multiple variations of the rule of 6; (ii) An electronic calculator to aid the prescribing process- this generated printed prescriptions and syringe labels; (iii) smart pump technology with an associated drug library, thus eliminating the use of the $\mathrm{ml} / \mathrm{hr}$ option on syringe pumps. The NICU clinical pharmacist provided training to NICU nurses and prescribers. PCIs were collected prospectively before and after implementation by the NICU clinical pharmacist. Descriptive statistics were generated for PCIs in the two time periods. Binary logistic regression analyses were used to examine the association between medication safety bundle implementation and the occurrence of PCIs (SPSS 23, IBM Corp.)

Results Prescriptions of HACIs were recorded on 178 days. The medication safety bundle was used on 56 days. A total of 191 patients required $\geq 1$ HACI. Prior to the implementation of the bundle, on review of 292 orders, there were 60 PCIs per 100 orders. After implementation, on review of 217 orders, there were 19 PCIs per 100 orders (OR 0.15 95\% Confidence Interval 0.10-0.23). Analyses adjusting for gestation at delivery, age, weight, staffing ratio and unit occupancy did not alter the findings. There was a large reduction in incomplete prescriptions and labels. A shift in the nature of PCIs was noted post implementation, with new issues related to selection of the wrong infusion routes or bypassing smart pump functionality.

Conclusion Implementation of this medication safety bundle was associated with a reduction in the number and a shift in the nature of PCIs associated with the prescribing, preparation and administration of HACIs. This study provides useful data to inform a future study to assess the effectiveness of this medication safety bundle on a broader scale.

\section{GP257 MORTALITY RISK AMONG VERY LOW BIRTH WEIGHT INFANTS BORN IN THE REPUBLIC OF IRELAND, 2014- 2016}

${ }^{1}$ Richard Corcoran, ${ }^{1}$ Linda Drummond, ${ }^{2}$ Anne Twomey ${ }^{*}$, ${ }^{3}$ Brendan Murphy, ${ }^{1}$ Richard Greene. 'University College Cork, Cork, Ireland; ${ }^{2}$ National Maternity Hospital, Dublin, Ireland; ${ }^{3}$ Cork University Maternity Hospital, Cork, Ireland

10.1136/archdischild-2019-epa.316
Background and aim Globally, there are approximately six million deaths of children under 5 years of age each year, the leading cause of which is preterm birth complications. Very preterm infants are at especially high risk of mortality. The Vermont Oxford Network (VON) is a non-profit voluntary collaboration of health care professionals from nearly 1000 neonatal intensive care units around the world and it maintains a database of information regarding the care and outcomes of high-risk newborn infants.

We aimed to use the VON data to assess whether very low birth weight (VLBW) infants born in Ireland in 2014-2016 had a higher than expected risk of death.

Methods Since 2014, all 19 neonatal units in Ireland have contributed data to the VON database on VLBW infants, defined as an infant who is born alive and whose birth weight is between 401 and 1500 grams OR whose gestational age is between 22 weeks 0 days and 29 weeks 6 days (inclusive). VON colleagues use multivariable logistic regression models to quantify the risk of mortality associated with a range of infant characteristics. We used coefficients from these regression models to calculate standardised mortality ratios (SMRs).

Results The VON database had data on 1,812 VLBW infants born in Ireland in 2014-2016, of which 1,765 were records from their hospital of birth. The mortality risk for these 1,765 infants was 1.17 times higher than expected, a statistically significant excess mortality (95\% CI: 1.05, 1.29). Infants born at 22-23 weeks had a 23\% higher mortality risk $(\mathrm{SMR}=1.23,95 \% \mathrm{CI}: 1.02,1.44)$ that was almost wholly due to the infants not administered resuscitation. Infants born at 24-27 weeks in a tertiary unit did not experience higher than expected mortality $(\mathrm{SMR}=1.01,95 \% \mathrm{CI}: 0.80,1.23)$ but those born in non-tertiary units had a $70 \%$ higher mortality risk $(\mathrm{SMR}=1.70,95 \% \mathrm{CI}: 1.25,2.15)$.

Conclusions These findings support the recommendations that resuscitation should be administered to all infants born at 23 weeks who present in favourable condition, i.e. without congenital anomaly, severely small for gestational age, severe hypoxia or severe infection. In line with the existing Model of Care for Neonatal Services in Ireland, infants born before reaching a gestational age of 28 weeksshould ideally be delivered at a tertiary neonatal unit.

\section{GP258 THE ROLE OF REFLECTIVE MATERIALS IN NEONATAL JAUNDICE - A PRE-CLINICAL STUDY}

Gearoid McGauran*, David Healy, Prof Eugene Dempsey. Cork University Maternity Hospital, Cork, Ireland

\subsection{6/archdischild-2019-epa.317}

Introduction Jaundice is a common reason for admission to neonatal units. The mainstay of treatment is phototherapy ${ }^{1}$. During phototherapy much of the light emitted fails to reach the baby's skin due to dispersion. It has been hypothesised that the use of reflective materials around the incubator may increase the efficacy of phototherapy with little added cost ${ }^{2}$. Aims This study aimed to determine whether the addition of reflective materials could increase irradiance during single-light phototherapy and whether any increase was comparable to addition of a second light source.

We also investigated whether the addition of reflective materials could increase irradiance during double-light phototherapy. 